\title{
Obstetric Anesthesia and Depression: Data Granularity and the Risk of Confounders
}

Stephanie Kent, MBBS; Danielle Eusuf, FRCA; Stephanie Harper, MBChB; Eleanor Tanqueray, MBChB; Clifford L. Shelton, PhD

To the editor -

We read Guglielminotti and Li's study ${ }^{1}$ of postpartum depression and anaesthetic mode for Cesarean delivery with great interest, and discussed it at our journal club at the North West School of Anaesthesia, UK. This observational study concluded that women had increased odds of postpartum depression, suicidal ideation and self-inflicted injury, when they underwent general anaesthesia (GA) for Cesarean delivery, compared with neuraxial anesthesia.

Although based on data acquired in New York State, this article received broad media attention in the UK; as a consequence of this, the Royal College of Anesthetists issued a statement ${ }^{2}$ expressing concern about the possibility of confounding factors. We share the concerns of the Royal College, in particular because the quality of the data available to this study limits the conclusions that can be drawn. We feel that Guglielminotti and Li could perhaps have been more forthright about the limitations of their study in the manuscript.

Firstly, the authors were unable to stratify their analysis by clinical urgency; an important omission considering that GA is typically provided for only the most urgent surgical deliveries, the indication for which (e.g. fetal hypoxia) may be independently associated with negative postnatal mental health outcomes. Indeed, the two meta-analyses ${ }^{3,4}$ cited by the authors in their introduction, linking cesarean section to increased risk of postpartum depression, both found an increased risk amongst mothers who underwent emergency Cesarean delivery, compared to those who received elective surgery.

Furthermore, because no information on neonatal outcome was available, this potential confounding factor, known to be associated with postpartum depression ${ }^{5}$, could not be accounted-for.

In the UK, Cesarean sections are classified according to a nationally-agreed system relating to clinical urgency. Category 1 indicates maternal or fetal compromise, with immediate threat to the life of the woman or fetus; category 2 indicates maternal or fetal compromise, with no immediate threat to life; category 3 indicates no maternal or fetal compromise, but a requirement for early delivery; category 4 indicates delivery at a time to suit the woman and maternity services (i.e. elective surgery). It can be seen from this that even within the broad category of 'emergency' Cesarean sections (categories 1-2) there is a wide variation of clinical urgency, and as such a wide variation in anaesthetic technique.

Analysis of the 2018-2019 data from Blackpool Victoria Hospital, Lancashire, UK indicates that 35\% of category 1 cesarean sections were performed under GA with a 9.2\% conversion from neuraxial anaesthesia; category 2 had 6\% GA rate with 5.4\% conversion; category 3 had a 4\% GA rate with 3\% conversion; and category 4 had $2 \%$ GA rate with $1.2 \%$ conversion (Table). These data are broadly consistent with previously-measured national trends in the UK .

The majority of GAs at Blackpool Victoria Hospital (43/66) were performed to facilitate rapid delivery in category 1 Cesarean sections due to urgent, potentially life-threatening circumstances; in the remainder of Cesarean sections (i.e. in non-life-threatening circumstances) the majority of GAs were performed due to failure to achieve a neuraxial block or intraoperative discomfort during neuraxial anaesthesia (Table). Although we do not have data on incidence of postpartum depression amongst our patients, it 
is plausible that any of these reasons for providing GA could contribute to postnatal mental health problems, yet they are not represented in Guglielminotti and Li's data.

Whilst we commend Guglielminotti and Li for contributing to the debate around mode of anaesthesia in obstetric practice, we believe that this study only superficially examines the surface of a complex issue, and further research is needed before practice (either in terms of mode of anaesthesia or the process of consent) can change. In order to avoid similar confounders in future studies, the reasons for the provision of GA should be known, as should the degree of urgency and the clinical outcomes of both mother and baby. In conclusion, despite the known risk of GA in the obstetric population, general anaesthesia will still remain an indispensable option in the most urgent of cesarean deliveries.

\section{Acknowledgments}

The authors wish to thank Dr Chris Dunkley for providing the data on anesthesia for cesarean delivery at Blackpool Victoria Hospital and acknowledge the contribution of Drs Sarah Thornton and Louise England in establishing the Self-IsolAting Virtual Education (SAVEd) project, to continue to provide education during the coronavirus disease 2019 (COVID-19) pandemic. 


\section{References}

1. Guglielminotti J., Li G. Exposure to General Anaesthesia for Cesarean Delivery and Odds of Severe Postpartum Depression Requiring Hospitalization. Jan 2020. Anesthesia \& Analgesia. In press - doi: 10.1213/ANE.0000000000004663.

2. https://rcoa.ac.uk/news/rcoa-response-exposure-general-anesthesia-cesarean-delivery-oddssevere-postpartum-depression, accessed 17/06/2020.

3. Moameri H., Ostadghaderi M., Khatooni E., Doosti-Irani A. Association of postpartum depression and cesarean section: A systematic review and meta-analysis. 2019. Clinical Epidemiology and Global Health. 7: $471-480$.

4. Xu H., Ding Y., Ma Y., Xin X., Zhang D. Cesarean section and risk of postpartum depression: a meta-analysis. 2017. Journal of psychosomatic research. 97: 118-126.

5. Vigod S., Villegas L., Dennis C-L, Ross L. Prevalence and risk factors for postpartum depression among women with preterm and low-birth-weight infants: a systematic review. 2010. British journal of obstetrics and gynaecology. 117: 540-550.

6. Obstetric Anaesthetists' Association, National Obstetric Anaesthesia Data for 2012 -A report, https://www.oaa-

anaes.ac.uk/assets/ managed/cms/files/NOAD\%20REPORTS/National\%200bstetric\%20Anaesth esia\%20Data\%20for\%202012\%20final.pdf, accessed 17/06/2020. 
Table: Cesarean delivery data from Blackpool Victoria Hospital, Blackpool, Lancashire, UK, from 20182019 , stratified by category of cesarean section and anaesthetic type

\begin{tabular}{|l|l|l|l|l|}
\hline Category of & Total & Neuraxial & General & Neuraxial \\
& & anaesthetic $(\%$ & anaesthetic $(\%$ & converted to GA \\
& & & total) & \\
& & & & neuraxial) \\
& & & \\
\hline Category 1 & 122 & $79(65 \%)$ & $43(35 \%)$ & $8(9.2 \%)$ \\
\hline Category 2 & 188 & $176(94 \%)$ & $12(6 \%)$ & $10(5.4 \%)$ \\
\hline Category 3 & 102 & $98(96 \%)$ & $4(4 \%)$ & $3(3.0 \%)$ \\
\hline Category 4 & 339 & $332(98 \%)$ & $7(2 \%)$ & $4(1.2 \%)$ \\
\hline Total & 751 & $685(91 \%)$ & $66(9 \%)$ & $25(3.5 \%)$ \\
\hline
\end{tabular}

${ }^{a}$ Cesarean sections carried out under neuraxial anaesthesia (converted anaesthetics not included)

${ }^{b}$ Cesarean sections carried out under general anaesthesia (converted anaesthetics included)

${ }^{\mathrm{C}}$ Cesarean sections carried out under GA due to failed neuraxial anaesthetic for any reason (\% of total attempted neuraxial) 